\title{
PROSPECTS FOR OCTOPUS RHODOPSIN UTILIZATION IN OPTICAL AND QUANTUM COMPUTATION
}

\author{
V. Sivozhelezov ${ }^{a}$, C. Nicolini ${ }^{a, b}$ \\ ${ }^{a}$ Fondazione Elba, Via delle Testuggini snc, Roma, Italy \\ ${ }^{b}$ Nanoworld Institute and Biophysics Chair, University of Genoa, Genoa, Italy
}

\begin{abstract}
Visual membranes of octopus, whose main component is the light-sensitive signal transducer octopus rhodopsin (octR), are extremely highly ordered, easily capture single photons, and are sensitive to light polarization, which shows their high potential for use as a QC detector. However, artificial membranes made of octR are neither highly enough ordered nor stable, while the bacterial homolog of octR, bacteriorhodopsin (bR), having the same topology as octR, forms both stable and ordered artificial membranes but lacks the optical properties important for optical QC. In this study, we investigate the structural basis for ordering of the two proteins in membranes in terms of crystallization behavior We compare atomic resolution 3D structures of octR and $\mathrm{bR}$ and show the possibility for structural $\mathrm{bR} / \mathrm{octR}$ interconversion by mutagenesis. We also show that the use of (nano)biotechnology can allow (1) high-precision manipulation of the light acceptor, retinal, including converting its surrounding into that of bacterial rhodopsin, the protein already used in optical-computation devices and (2) development of multicomponent and highly regular 2D structures with a high potential for being efficient optical QC detectors.
\end{abstract}

Визуальные мембраны осьминога, основным компонентом которых является светочувствительный трансдюсер, родопсин осьминога (octR), отличаются высокой упорядоченностью, возможностью детектировать единичные фотоны и чувствительностью к поляризации света, таким образом обладая большим потенциалом для использования в качестве QC-детекторов. Однако искусственные мембраны из octR недостаточно стабильны и недостаточно упорядоченны, в то время как имеется бактериальный гомолог octR - бактериородопсин (bR), топология которого идентична топологии octR. Бактериородопсин образует стабильные и высокоупорядоченные искусственные мембраны, но не имеет оптических свойств, важных для оптических QС. В настоящей работе исследуются структурные основы упорядоченности octR и $\mathrm{bR}$ в мембранах в связи с кристаллизуемостью этих белков. Сравнение пространственных структур octR и $\mathrm{bR}$ показало, что преобразование структур octR в bR и обратно возможно путем направленного мутагенеза. В работе также показано, что использование (нано)биотехнологии может позволить 1) высокоточные манипуляции с акцептором светового кванта octR, ретиналем, в том числе замену его окружения на соответствующее окружение $\mathrm{bR}$, и 2) разработку многокомпонентных упорядоченных двумерных структур с возможностью использования последних в качестве эффективных оптических QC-детекторов.

\section{INTRODUCTION}

Optical implementations of qubits were successfully applied in a wide range of theoretical and experimental quantum computation research, as well as applications, due to intrinsic lack of decoherence and ability for high-precision control [1]. However, highly efficient singlephoton detectors are required for displaying the result of optical QC because of the extremely 
small photon-photon coupling available in existing materials [2]. The visual membranes of higher biological organisms easily capture single photons [3], while in some biological species they also capture the polarization of light [4], the latter being the signal-encoding parameter in optical QC. The extremely high ordering of octopus visual membranes, as well as its ability to capture single photons and sensitivity to light polarization, suggest the possible use of rhodopsins, the main component of such membranes, as a material for photonic qubit detector. Octopus rhodopsin (octR), a typical example of light transducer in deep-water species that need extreme photosensitivity, including sensitivity to polarization of light, is exceptionally well ordered in its membranes in vivo. However, artificial membranes of octR are practically liquid as those made of their mammalian homologs. So far they have neither 3D structures nor suitable crystals. In 2D, squid rhodopsin, a close homolog of octR, forms 2D crystals similar to those of bovine rhodopsin, allowing one to obtain only approximate structures from crude (8 ̊̊ resolution) X-ray density map [5].

In contrast, bacteriorhodopsin (bR) has already a huge body of such structure/function data (reviewed in [6]) with respect to bR, as well as nanotechnology applications, e.g., [7], because it forms extremely stable and well-ordered structures.

Functionally, octR is a light transducer which captures single light quanta, while bR is a light harvester which does not require high sensitivity.

Structurally, octR and bR belong to the structural superfamily of rhodopsin-like proteins sharing the overall seven transmembrane helix topology of BR except for some details in distances and relative orientations of the helices [8]. This allows one to use bR as the primary template for all structure/function studies.

Similarities and differences between visual rhodopsins and bacteriorhodopsins are extensively discussed elsewhere [9], mainly using bovR as an example. Less attention has been paid to cephalopod rhodopsins, in which rhodopsin is the main component of microvillar membranes, microvilli being cylindrical extensions of the cell membrane, arranged hexagonally within the rhabdome (photoreceptor) [10].

As the first step, we build a 3D model of octR using bovR as a template, and compare it with the structure of $\mathrm{bR}$. We also discuss this comparison in terms of nanotechnology applications that have been reported for bR [31] but can also be implemented in octR. We also built a model for mutant protein aimed at ocrR/bR interconversion. In this work, we decided to explore only possible mutations in the immediate surroundings of the retinal, while mutations affecting the overall fold will be addressed elsewhere.

\section{METHODS}

The homology modeling was performed using the SwissModel suite of programs, using the DeepView program (www.expasy.org/spdbv) as the front end. The tools PCAPPS version 2 (darwin.nmsu.edu/cgi-bin/pcapss_v2.cgi) and Porter [11] were used for selecting the template and calculation of octR secondary structure, respectively. Optimization of the obtained model with the retinal included was done using the evaluation version of the package HyperChem v.7.0 (www.hyper.com), using the parameters of the AMBER force field [12].

Since partial charges of the retinal are highly dependent on the environment, the following combination of quantum mechanical and molecular mechanical (QM/MM) calculations was adopted: 
Step 1: the geometry of opsin was MM-optimized for the uncharged and fixed retinal;

Step 2: retinal charges were calculated using QM (PM3 parametrization);

Step 3: the entire octR molecule was MM-optimized; Go to Step 2. Iterations continued until convergence. The 3D structures of models obtained were compared to those available in PDB using the VAST tool [13].

\section{RESULTS AND DISCUSSION}

Selecting a Tool for Modeling the 3D Structure of octR. For a transmembrane GPCR protein like octR, this is not a trivial task. While classical homology modeling is still successfully used [14], two other approaches were implemented, one based on intraprotein hydrogen-bond optimization [15] and the other based on first principles of transmembrane protein assembly [16].

As for the hydrogen-bond optimization approach, it seems to be working well if experimentally derived constraints are used, but the experimental data underlying these constraints are themselves under discussion for octR. Besides, the presence of retinal within the transmembrane part of the molecule could hamper correct hydrogen bond assignment which is crucial for accuracy of the method.

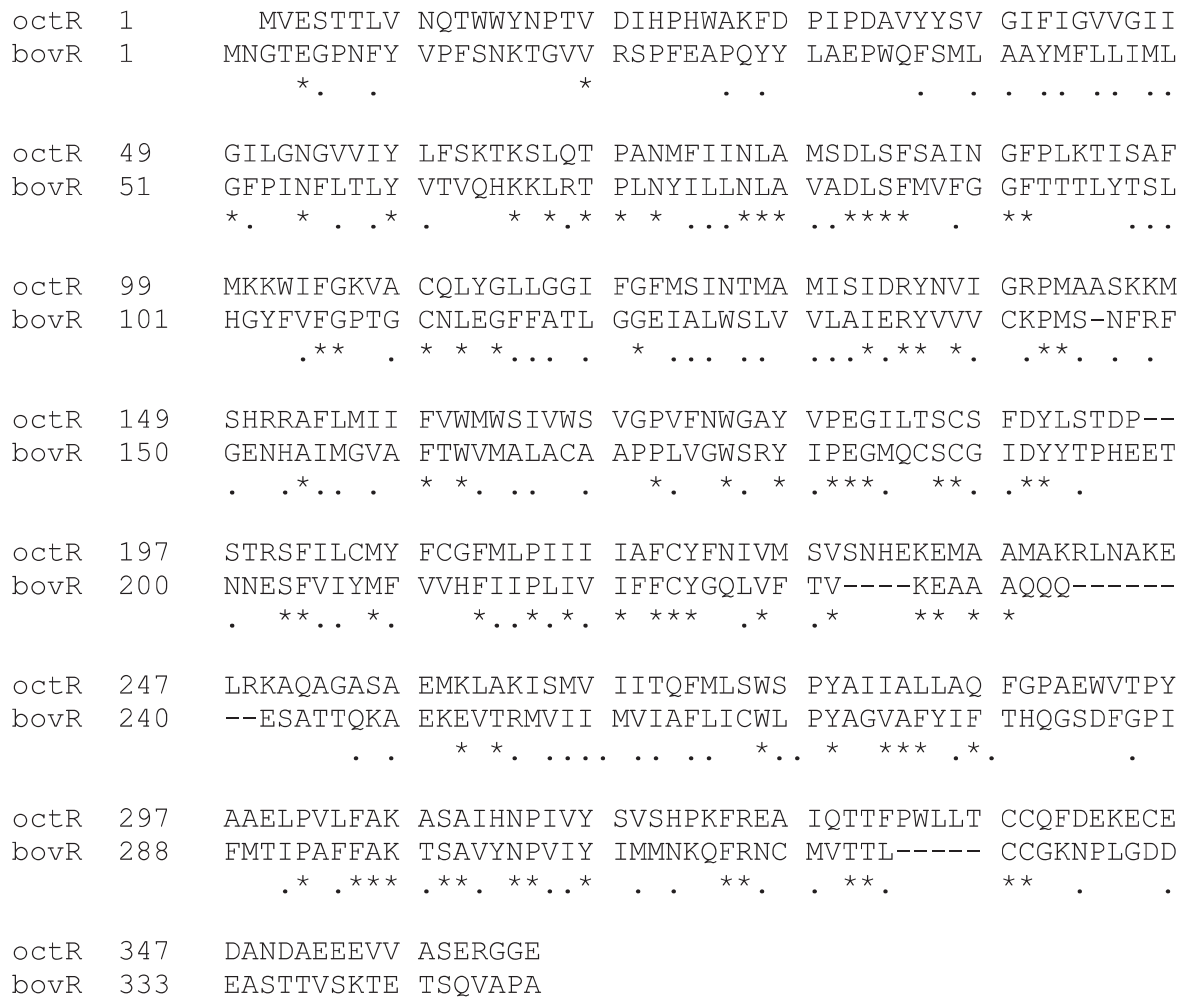

Fig. 1. Alignment of octopus rhodopsin versus the bovine rhodopsin used in building the homology model. Identical residues are marked with an asterisk, similar with a dot 
The first principles approach is more attractive considering also that it has already been tested on bovine rhodopsin. Since it is mostly based on assigning the transmembrane helices, we started by predicting positions of the helices and then manually adjusting the resulting alignment of octR versus bR using sequence identity and similarity. The eventual alignment is shown in Fig. 1. The resulting approach is therefore a hybrid between homology modeling and first-principles modeling. The template selected for homology modeling was the PDB entry 1U19, and the resulting model is shown in Fig. 2.

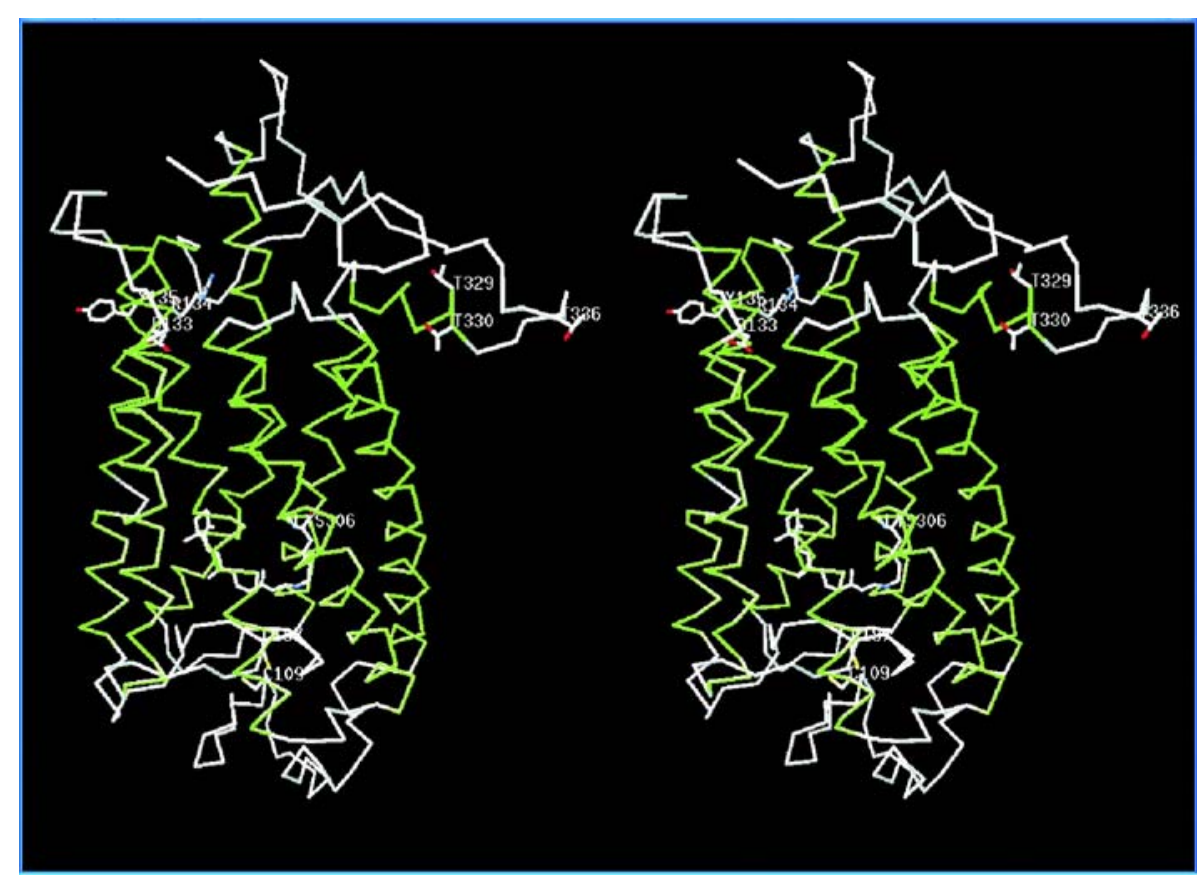

Fig. 2. Stereo view of the homology model of octopus rhodopsin as a $\mathrm{C} \alpha$ trace, with the retinal shown as sticks. Invariant residues are numbered. Alpha helices are shown in green

Model Quality Estimated by Comparison of the Model with Its Template. One can see that the model reproduces the positions of the residues surrounding the chromophore (retinal) correctly, particularly the Lys306 residue providing the Schiff base connection of the protein to the retinal. Notably, the model shows higher alpha helical content than its template (203 versus 190 alpha helical residues), a rare occasion in comparative modeling. This could be a basis for higher organization of octR into 2D arrays compared to the bovR, but the experimental data [5] show that it happens only in vivo but not in vitro. Definitely some factor other than the rhodopsin structure itself is responsible for the high ordering of octR in vitro, and, as further analysis will suggest, this could be interaction with another transmembrane protein present in membranes together with octR.

The sequence identity level in the eventual model was $24.4 \%$, which is above the average $20 \%$ quoted for comparisons of vertebrate versus invertebrate visual pigments and therefore supports the reliability of our model. Since all visual pigments described to date have distinc- 


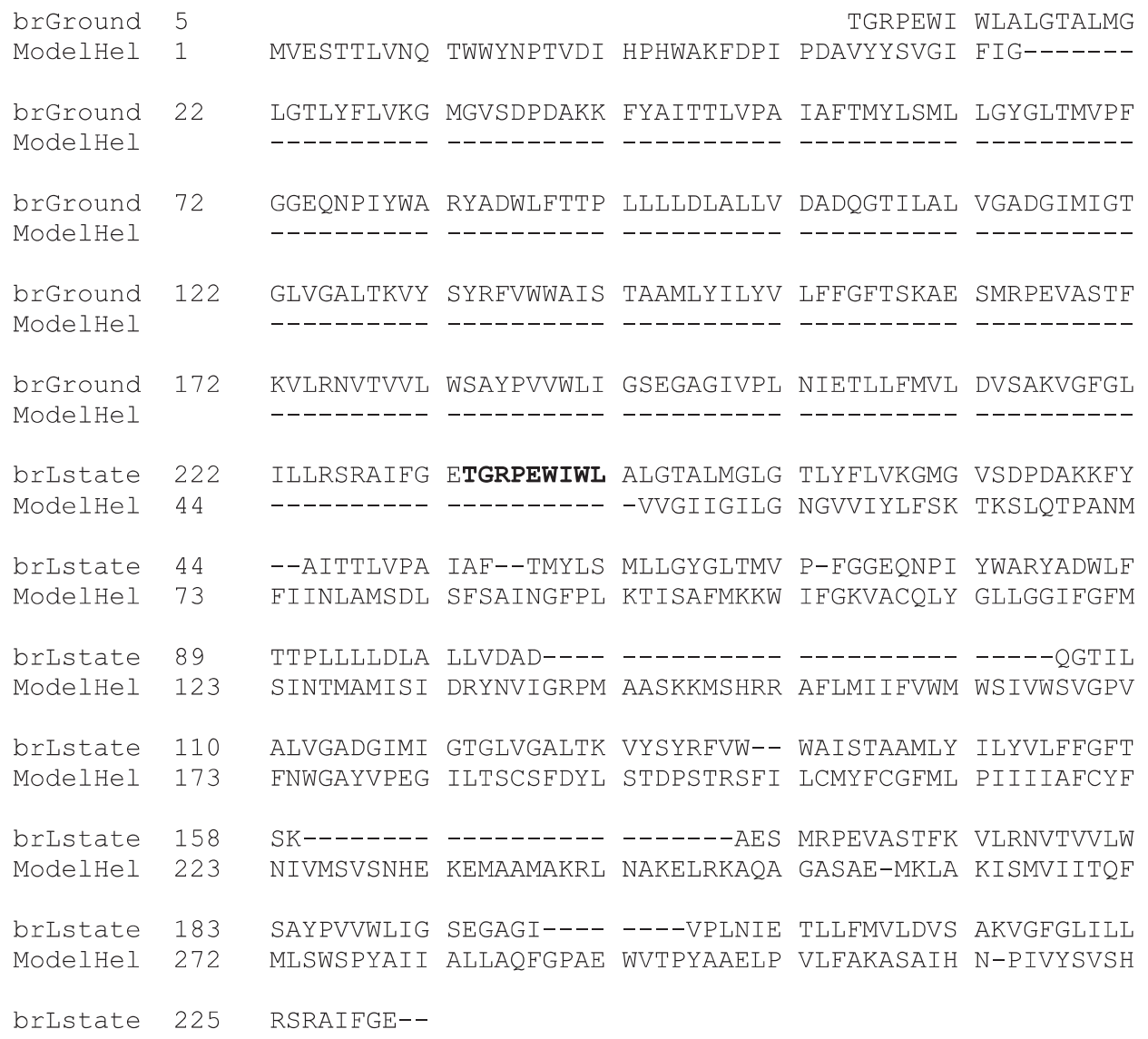

Fig. 3. Structural alignment of octopus rhodopsin with the ground state and the L state of bacteriorhodopsin. The start of the L-state sequence is marked in bold

tive features [17], quality of the model can be assessed by the model's ability to reproduce those features. The first is a lysine in the middle of the seventh putative transmembrane segment, corresponding to Lys-296 in bovine rhodopsin, which is the site of covalent binding of the chromophore via a retinylidene Schiff base. In our case, it is Lys306. The next feature is a pair of cysteines corresponding to Cys-110 and Cys-187 in bovine rhodopsin, which are presumed to form a disulfide bond connecting the first and second extracellular loops. These are present in our model as Cys109 and Cys187. Another «quality indicator» is presence of the sequence (Glu/Asp)-Arg-Tyr, or a close match to this sequence, at the beginning of the second cytosolic loop, in our case it is Asp133, Arg134, and Tyr135. Finally, one or more serine or threonine residues should be located in the cytosolic carboxyl terminus, which in bovR are the sites of light-dependent phosphorylation by rhodopsin kinase. In our model, those are threonines 329,330 , and 336 .

Identity of the counterion is another important issue both with respect to both the structure/function relationships and nanotechnology applications. Indeed, in both octR and bR, the 
conformational rearrangements required for function (proton pumping for bR, signal transduction for octR) demand precedent storage of energy ([9] and references therein), which is in both cases achieved by separating the charge of the retinal/lysine Schiff base from its counterion, acting also as a starting point for proton pumping in bR. Our model predicts that the position equivalent to the bovR counterion (Glu113) is occupied by Tyr112. This tyrosine always remains neutral [18] so its role as the counterion is ruled out. However, our model predicts that the residue Glu181 is within $5 \AA$ of the Schiff base nitrogen and can therefore serve as the counterion. This is in perfect agreement with the recent mutational analyses proving that it is the Glu181 that is the counterion in invertebrate rhodopsins [19] while the vertebrate-rhodopsin counterion (Glu113 in bovR) was acquired later in the course of evolution.

Comparison of the octR Model with $b R$ and Mutations Suggested for octR/bR Interconversion. Considering that the ground-state isomers of retinal are 11-cis for octR and all-trans in $\mathrm{bR}$, we attempted to accommodate an all-trans retinal in the octopus opsin by means of molecular modeling. We tried to introduce all-trans retinal into a new cavity in which it would have the same surroundings as in bR. The latter was done by introducing putative mutations. The resulting mutant octR should possess similar properties to bR, at least in the immediate vicinity of the retinal environment. The mutations suggested to graft the bR-like retinal binding pocket to octR are Gly120Trp, Phe121Met, Ile124Trp, Gly209Ser, Tyr278Trp, Val302Lys, Ala305Tyr, and Lys306Phe. The resulting retinal-binding pocket in the putative mutant is shown in Fig. 4.

One can see that no mutation is required to insert the counterion into octR at the spatially equivalent position of bR. Indeed, the Asp 83 of bR and Glu181 of OctR are less than $3 \AA$ apart. The mutation Gly120Trp is required to insert a tryptophan residue at the position identical to that of Trp182 of bR. The Trp182 is known to be responsible for the functionally important interaction with the retinal 9-methyl group in the L intermediate [20]. The mutation Phe121Met in octR reproduces the Met145 of bR, for which computer simulations predict involvement in the binding pathway of retinal [21]. The mutation Ile124Trp introduces a tryptophan equivalent to Trp138 in bR, which is involved in a functionally important pi-cloud H-bond with Trp189 in bR [22]. The Gly209Ser provides the bR equivalent of Ser141. The Tyr278Trp is designed to provide the equivalent of Trp189 of bR, which is also involved in hydrogen bind with Tyr83 [21] and exhibits marked UV Raman spectroscopy-detected changes in its environment during the photocycle [22]. The Val302Lys and Lys306Phe are the crucial mutations shifting the retinal attachment site. The Lys302 now provides the Schiff base link to the retinal, while Phe306 should block the retinal isomerization to 11-cis. The Ala305Tyr mutation is introduced to mimic the bR residue Tyr185. The remaining residues neighboring the retinal already have their structural counterparts in the native octR and require no mutation. One can see that three new tryptophan residues are proposed to be introduced in the mutant octR. At a first glance, the bulky tryptophan residues could destabilize the structure. However, this notion comes from experience with soluble proteins where tryptophans could destabilize the hydrophobic core, which does not exist in transmembrane proteins like rhodopsins. Besides, recent NMR estimates of main chain and side chain mobility in bovR [23] show that the tryptophan side chains are even less mobile than backbone atoms, so most likely the backbone atoms can adapt their conformations to the new tryptophans.

According to our model, the mutations proposed for moving the Schiff base linkage to another position should result in absorbance spectrum changes, as well as change of the time 


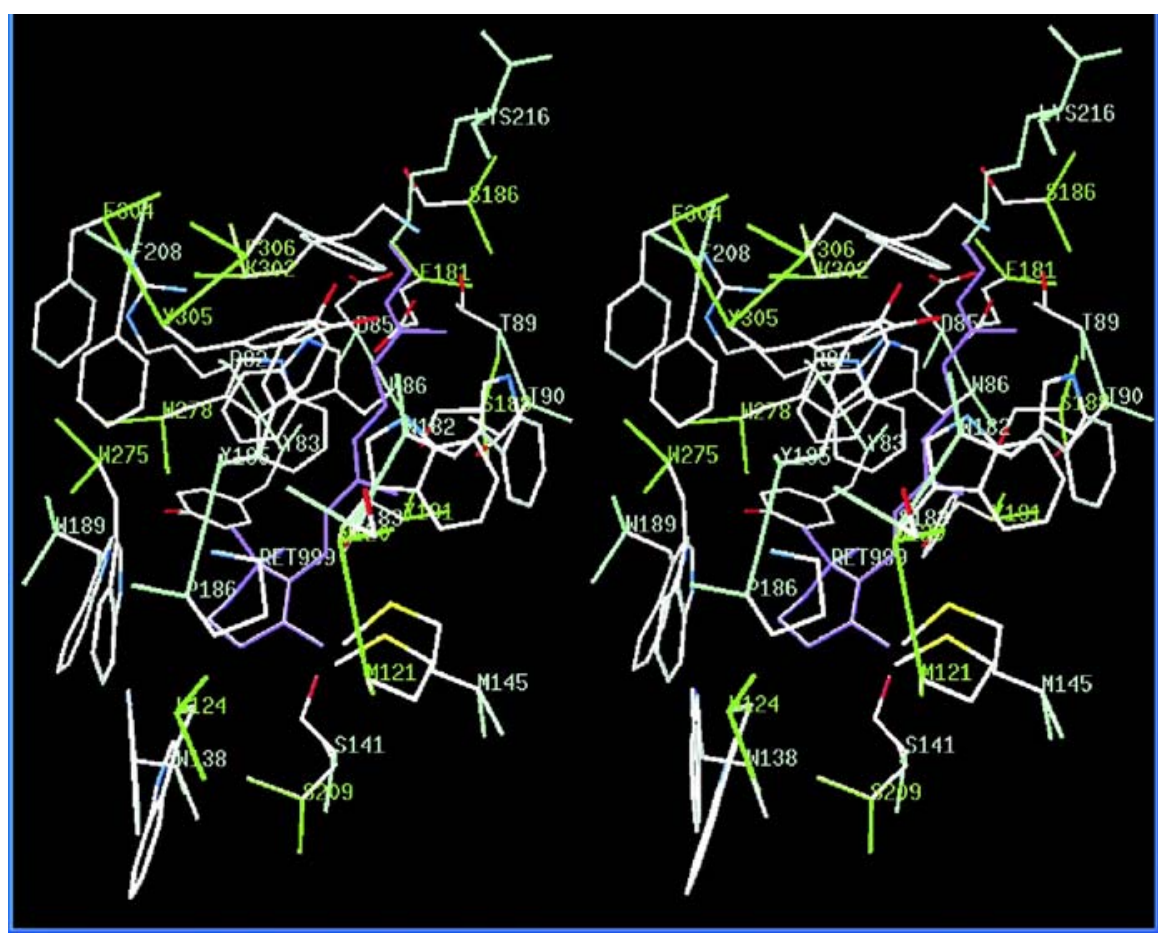

Fig. 4. Stereo view of the retinal-binding pocket in the putative mutant octopus rhodopsin superimposed on the retinal pocket of bacteriorhodopsin. Backbones of the octR and bR as well as residue numbers are in green and cyan, respectivevly. Side chains are CPK-colored

scale of the earliest photoprocess detected. The corresponding experimental techniques are described in [9]. The mutations introducing the tryptophans should be verified by observation of decreased mobility of the nearby parts of the protein main chain, according to the NMR technique of [24].

Binding of All-trans Retinal to Octopus Opsin. To find out if the native octR could accommodate retinal which is the excited state for octR but the groud state in bR, we inserted all-trans retinal into the native octR retinal cavity. This allowed checking if octR could bind all-trans retinal in the same way it binds its native ground-state 11-cis retinal. These very simplified strategies are dictated primarily by the approximate nature of homology modeling.

As follows from Fig. 5, octopus opsin should readily recombinate with all-trans retinal. The superimposed structures allow one to see that octR opsin can accommodate retinal both in its native 11-cis conformation and in the all-trans conformation. This is supported by the experimental data [25] showing that, while both bovR and octR are easily regenerated with their native 11-cis retinal, octR can be additionally regenerated with all-trans retinal and moreover the 13-cis retinal which is the native ground-state chromophore for bR. It is further supported by the recent finding that rhodopsin from lancelet (amphioxus), a primitive chordate, is, like octR and unlike bovR, able to bind all-trans retinal, and mutational analyses revealed that Trp265 is responsible for this property [26]. We found that the pairwise homologies 


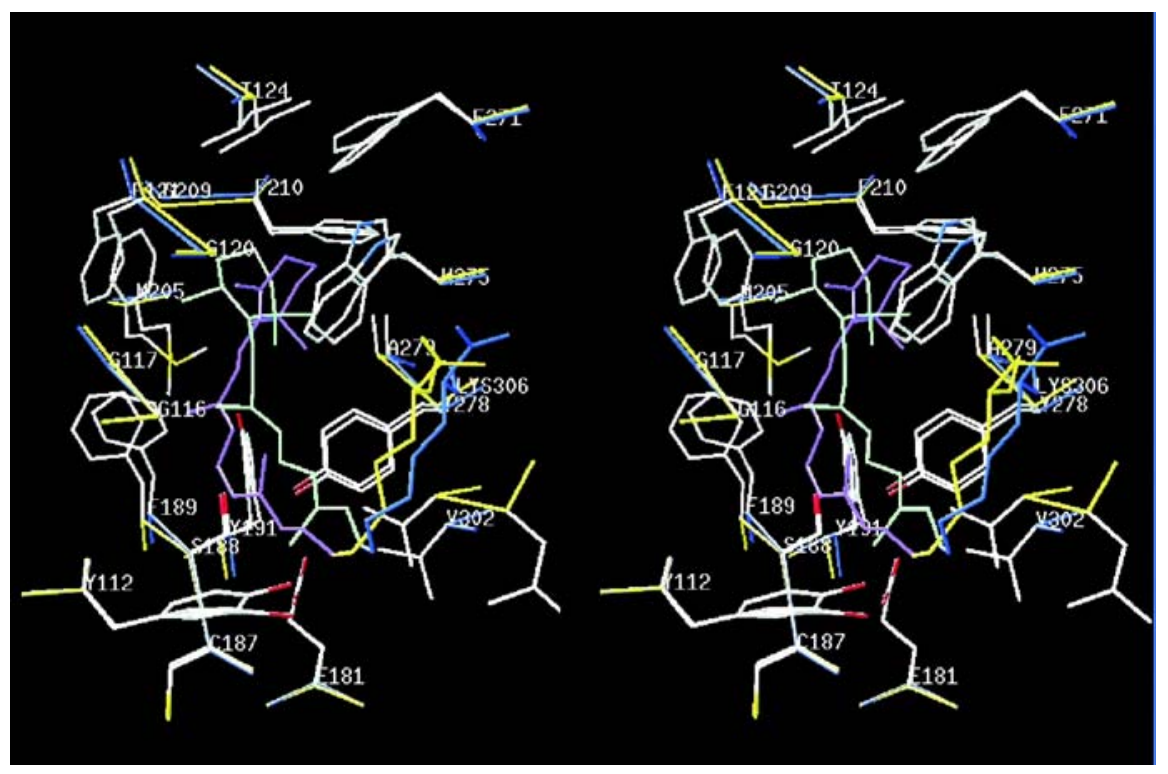

Fig. 5. Stereo view of the superimposed retinal-binding pocket for 11-cis (left) and all-trans (right) retinal. The retinal is colored in magenta and cyan respectively, backbone atoms in yellow and blue respectively, and side-chain atoms according to the CPK scheme

between rhodopsins from lancelet and octopus, as well as bovine and octopus rhodopsins, are similar (about $30 \%$ identity) but the 275 th amino acid position in our octR model equivalent to the 265th position of lancelet rhodopsin is also occupied by tryptophan (data not shown). In contrast, there are no tryptophans at the corresponding position of bovine rhodopsin or positions close in its amino acid sequence. On the one hand, this adds to the validity of our octR model since it predicts not only the experimentally known effect, i.e., the ability of octR to bind all-trans retinal, but also its cause, i.e., the tryptophan at the 275 th position. On the other hand, none of the mutations proposed in this paper is at the 275 th position, so they are unlikely to affect this property. Besides, the mutation of the neighboring Tyr278 to Trp proposed herein is unlikely to affect the conformation of Trp275 because both Tyr and Trp residues have aromatic side chains.

However, the conformations of the ionone ring in the model of all-trans reconstituted octR and the 11-cis dark-state octR are not easily interconvertible within the protein because of contacts with protein side chains. This is supported by the fact that intraprotein conversion of 11-cis to all-trans conformation in any rhodopsin requires the entire range of the photoprocess, which has the time scale of milliseconds. Upon activation by light, followed by transition through batho- and meso- to limi-rhodopsin, the retinal molecule dissociates from octR to form the free retinal and the octopus opsin, so octR regenerates again only after the retinal has been conversed into the 11-cis configuration. However, our data, together with the data of [25], as well as evidence for presence of alternative binding sites for retinal on opsin [27], suggest that, unless the all-trans retinal is quickly transformed into 11-cis retinal upon release into the lipidic matrix or bound to some transporter protein delivering it to and from the 
isomerization site, it will be very likely sequestered by the same octopus opsin, thus leading to the inactive form of octR thereby terminating the visual cycle. In reality, the visual cycle is not terminated, which implies that the all-trans retinal release from octR is followed by its uptake by a protein other than rhodopsin.

One such protein could be the retinochrome, the retinal isomerization enzyme (Molina et al., 1992). However, the body of the experimental data available for invertebrate rhodopsins suggest that the retinochrome is located away from the rhodopsin, but linked to it via a shuttle protein RALBP [28]. We will now consider the distance from the site of retinal release from octR at which retinal uptake by RALBP must take place. Assume the excessively high diffusion coefficient of $D=10^{-7} \mathrm{~cm}^{2} / \mathrm{s}$ for retinal in the membrane. Assume also that the time available for the all-trans retinal before it is sequestered by the octopus opsin as $t=10^{-7} \mathrm{~s}$, again unrealistically big because such a process should be limited by side-chain and main-chain torsional motions of the opsin and/or torsional motions of the retinal, all of which are at the nanosecond to picosecond time scale. Then, the maximal distance to which such «free» all-trans retinal can travel will be given by the Einstein-Smoluchowski equation $d=(2 D t)^{1 / 2} \sim 1.5 \cdot 10^{-7} \mathrm{~cm}$, which is only $15 \AA$ A. Therefore, the RALBP protein must be located very near the rhodopsin. Importantly, in other invertebrates like honeybee, such a retinal-binding protein has also been detected and moreover is able to perform the function of retinochrome [29].

\section{CONCLUSIONS}

Our calculations suggest that mutagenesis can practically arbitrarily be used to modify the immediate surroundings of the octR's photosensitive molecule, retinal, thus allowing one to graft the properties of $\mathrm{bR}$ to octR and vice versa. This is mostly done by incorporating tryptophans into the vicinity of the retinal, in the same way increasing the protein stability.

In terms of the application of octR in quantum or optical computations, this work has explored only one of two possible directions, namely, increasing stability and crystallizability of the octR-based systems that are intrinsically highly sensitive light sensors. The other option, that is, grafting the properties of octR to the already stable and crystallizable protein, remains to be explored.

The nature of stability of octR in vivo, however, is probably caused by the presence of another protein in addition to octR (possibly RALBP) which should be located in the immediate vicinity to octR in vivo. The presence of this protein can yield better stability and ordering of the octR-based assemblies, including crystals. Obtaining such structures containing rhodopsin and the other retinal-binding protein will give rise to a novel type of optical computation device. Indeed, the key property of bR allowing one to utilize it in optical memory device [7] is its existence in two stable forms. The same property is utilized in the existing optical computer [30] in which two lasers are used to encode signals on bR films, so that the signals can be added and subtracted according to the trinary logic system where positive unity, negative unity, and zero are designated by high concentration of purple form, high concentration of yellow form, and equal amounts of the two forms, respectively. Obviously this device is purely passive. If regularly structured octR/retinochrome films are obtained, they will contain two different proteins that can be manipulated independently. This will open the road to a new formulation of classical optical computing, now including 
active optical elements. Considering that the power of nanobiotechnology allows atomic level manipulation of proteins, this could allow embedding quantum computation units into the rhodopsin-based active computation media.

\section{REFERENCES}

1. US Government Advanced Research and Development Activity. QIST Quantum Computing Roadmap. Overview. P. 12; http://qist.lanl.gov. 2004.

2. Duan L.M., Kimble H.J. Scalable Photonic Quantum Computation through Cavity-Assisted Interactions // Phys. Rev. Lett. 2004. V.92. P. 127902.

3. Field G.D., Sampath A.P., Rieke F. Retinal Processing near Absolute Threshold: From Behavior to Mechanism // Ann. Rev. Physiol. 2005. V.67. P. 491-514.

4. Shashar N., Cronin T.W. Polarization Contrast Vision in Octopus // J. Exp. Biol. 1996. V. 199. P. 999-1004.

5. Davies A. et al. Three-Dimensional Structure of an Invertebrate Rhodopsin and Basis for Ordered Alignment in the Photoreceptor Membrane // J. Mol. Biol. 2001. V.314. P. 455-463.

6. Hirai T., Subramaniam S. Structural Insights into the Mechanism of Proton Pumping by Bacteriorhodopsin // FEBS Lett. 2003. V.545. P. 2-8.

7. Fischer T., Hampp N. A. Encapsulation of Purple Membrane Patches into Polymeric Nanofibers by Electrospinning // IEEE Trans. Nanobiosci. 2004. V.3. P. 118-120.

8. Faulon J.L., Sale K., Young M. Exploring the Conformational Space of Membrane Protein Folds Matching Distance Constraints // Protein Sci. 2003. V. 12. P. 1750-1761.

9. Bryl K. Visual and Archaeal Rhodopsins: Similarities, Differences and Controversy // Cell. Mol. Biol. Lett. 2003. V. 8. P. 285-296.

10. Pepe I. M., Cugnoli C. Retinal Photoisomerase: Role in Invertebrate Visual Cells // J. Photochem. Photobiol. B. 1992. V. 13. P. 5-17.

11. Pollastri G., McLysaght A. Porter: A New, Accurate Server for Protein Secondary Structure Prediction // Bioinformatics. 2004.

12. Wang J. et al. Development and Testing of a General Amber Force Field // J. Comput. Chem. 2004. V. 25. P. $1157-1174$

13. Madej T., Gibrat J. F., Bryant S. H. Threading a Database of Protein Cores // Proteins. 1995. V. 23. P. 356-369.

14. Miedlich S. U. et al. Homology Modeling of the Transmembrane Domain of the Human Calcium Sensing Receptor and Localization of an Allosteric Binding Site // J. Biol. Chem. 2004. V.279. P. 7254-7263.

15. Pogozheva I.D., Lomize A.L., Mosberg H.I. The Transmembrane 7-Alpha-Bundle of Rhodopsin: Distance Geometry Calculations with Hydrogen Bonding Constraints // Biophys. J. 1997. V.72. P. 1963-1985. 
16. Trabanino R. J. et al. First Principles Predictions of the Structure and Function of G-Protein-Coupled Receptors: Validation for Bovine Rhodopsin // Biophys. J. 2004. V. 86. P. 1904-1921.

17. Nathans J. Rhodopsin: Structure, Function, and Genetics // Biochemistry. 1992. V.31. P.49234931.

18. Nakagawa M. et al. How Vertebrate and Invertebrate Visual Pigments Differ in Their Mechanism of Photoactivation // Proc. Nat. Acad. Sci. USA. 1999. V.96. P. 6189-6192.

19. Terakita A. et al. Counterion Displacement in the Molecular Evolution of the Rhodopsin Family // Nat. Struct. Mol. Biol. 2004. V. 11. P. 284-289.

20. Yamazaki Y. et al. Interaction of Tryptophan-182 with the Retinal 9-Methyl Group in the L Intermediate of Bacteriorhodopsin // Biochemistry. 1995. V.34. P.577-582.

21. Isralewitz B., Izrailev S., Schulten K. Binding Pathway of Retinal to Bacterioopsin: A Prediction by Molecular Dynamics Simulations // Biophys. J. 1997. V.73. P. 2972-2979.

22. Rouhani S. et al. Crystal Structure of the D85S Mutant of Bacteriorhodopsin: Model of an O-like Photocycle Intermediate // J. Mol. Biol. 2001. V.313. P. 615-628.

23. Imasheva E. S. et al. Exploring the Function of Tyr83 in Bacteriorhodopsin: Features of the Y83F and Y83N Mutants // Biochemistry. 2001. V.40. P. 13320-13330.

24. Klein-Seetharaman J. et al. Differential Dynamics in the G-Protein-Coupled Receptor Rhodopsin Revealed by Solution NMR // Proc. Nat. Acad. Sci. USA. 2004. V. 101. P. 3409-3413.

25. Koutalos Y. et al. Regeneration of Bovine and Octopus Opsins In Situ with Natural and Artificial Retinals // Biochemistry. 1989. V.28. P. 2732-2739.

26. Tsukamoto H., Terakita A., Shichida Y. A Rhodopsin Exhibiting Binding Ability to Agonist Alltrans-Retinal // Proc. Nat. Acad. Sci. USA. 2005. V. 102. P. 6303-6309.

27. Schadel S. A. et al. Ligand Channeling within a G-Protein-Coupled Receptor. The Entry and Exit of Retinals in Native Opsin // J. Biol. Chem. 2003. V. 278. P. 24896-24903.

28. Terakita A., Hara R., Hara T. Retinal-Binding Protein as a Shuttle for Retinal in the RhodopsinRetinochrome System of the Squid Visual Cells // Vision Res. 1989. V.29. P. 639-652.

29. Pepe I. M., Cugnoli C., Schwemer J. Rhodopsin Reconstitution in Bleached Rod Outer Segment Membranes in the Presence of a Retinal-Binding Protein from the Honeybee // FEBS Lett. 1990. V. 268. P. 177-179.

30. Lewis A. et al. Optical Computation with Negative Light Intensity with a Plastic Bacteriorhodopsin Film // Science. 1997. V. 275. P. 1462-1464.

31. Nikolini C. et al. Towards Light-Addressable Transducer Bacteriorhodopsin Based // Nanotechnology. 1998. V.9. P. 223-227. 